\title{
Inverse Interpolation Techniques for Temporary Specifications Translation to Frequency Domain
}

\author{
Javier Joglar Alcubilla \\ Avionics Department, Barajas Institute, Avda. América 119, 28042, Madrid, Spain \\ jjoglar@educa.madrid.org
}

\begin{abstract}
It is proposed a translation methodology applied to temporal transient response characteristics to obtain parameters of the typical second order transfer function, in the frequency domain. This method based on automatic successive approximations, applying an inverse interpolation, will minimize overdesign and manual iteration in the process of searching for a controller in frequency. For each type of requirement, specific techniques are recommended to obtain specification translation, using the "ascending and descending differences Newton's procedure" due to its practice advantages, compared to other interpolation procedures. It will be used as interpolation functions those describing step signal or pulse signal, with subcritical damping and approached with a second order transfer function.
\end{abstract}

Keywords: Temporary specifications translation, frequency domain, inverse interpolation, quantitative feedback theory, tracking, sensitivity.

\section{Introduction}

In the technical design of controllers working in the frequency domain, as in QFT [9], the design specifications represent fulfillment requirements associated with the dynamics of the system to control in closed loop. These specifications or requirements are usually expressed in the time domain using temporary transient response characteristics [10], such as:

- The rise time " $t_{r}$ " or " $t r$ ", the maximum overshoot " $M p$ ", the maximum peak time " $t_{M p}$ ", the settling time " $t s$ " or " $t s$ " and settlement channel size or maximum permissible tolerance "dev", typical of the response of a closed loop control system to a step change in the input.

- The maximum peak value " $f_{\max }$ ", peak times " $t_{p}$ ", settling time " $t_{s}$ " or " $t s$ " and settlement channel "dev", associated with the response of a control system in closed loop disturbance rejection.

The responses to these changes, unitary step or disturbance represented by unitary pulse, can be approximated well by the responses of underdamped second order systems of step or pulse, respectively, which are formally described based on the parameters, damping coefficient " $\zeta$ " and natural frequency " $\omega_{n}$ " [21].

If you start with time domain specifications and design stage of the control methodology used is performed in frequency, the initial requirements imposed must be transformed to the frequency domain. It is seeking transfer functions in the frequency domain that satisfy the envelope limits, upper and/or lower, generated by the temporary specification. Traditional methodologies, as described in [4], the technique in [17] or the model-based procedure used in [3], generally start with second order transfer functions, using different parameters of frequencies $\omega_{n}$ and coefficients $\zeta$; adding poles and zeros to the function, you get to go closer as you can to the original temporal specification. But in any case, all procedures are based 
on trial and error manual iteration, which ends when the designer decides that the function obtained is adequate enough.

When you are talking about specifications translation from time domain to the frequency domain, actually you are not meaning to translate characteristics of the transient response $(t r, t s, M P, .$.$) into specifications on the frequency response (gain$ margin and phase, bandwidth, $\mathrm{M}$ circles, ..). It is using the nomenclature, widely used by authors, as in [24] or [10], to reference the control stage that deals with the determination of any transfer function in the frequency domain, which provides an equivalent response to the given by temporary requirements imposed.

There are other ways to simplify the specifications transfer technique [21]:

- Use classical hypothesis approximation of settling times; but only valid for tracking step inputs and settlement channels with $2 \%$ or $5 \%$ of the steady output value.

- Graphically, using abacuses.

A more accurate method which minimizes manual iteration and related overdesign problems [8], consists of using successive approximations to obtain the characteristics of transient response required, associated to specific parameters $\omega_{n}$ and $\zeta$. This is possible because if you observe the formal description of the responses step type or pulse approximated to a second order system, you can see that they are doubly transcendental equations in the desired time characteristic (settlement or rise), respect to $\omega_{n}$ and $\zeta$. A particular method is presented in [15], for temporary specifications transfer tracking to obtain the lower frequency limit, described in the form of transfer functions with underdamped second order response. This method, specific for tracking requirements, uses inverse interpolation techniques described in this paper for step type responses.

The aim of this work is to find techniques that provide transfer functions of second order in the frequency domain, whose responses are equivalent to those provided by the specifications imposed in the time domain. Its application in the design stage of frequency controllers must ensure a reduced overdesign and minimal iteration in the controller search process, for purposes of compliance with these temporal specifications and frequency transferred too.

This paper is the continuation of the study begun to achieve the goal described above in Spanish in [16].

\section{Temporary Specifications Translation to the Frequency Domain}

The importance of having a precise, automatic and specific methodology to translate temporary specifications to frequency is obvious, especially in the final stage of system responses analysis in control techniques, where the controller design is done in the frequency domain [13]. With a precise temporary specification transfer to the frequency domain, the specifications compliance in frequency will also mean fulfilling of them in time with a higher probability. Thus, the necessary degree of manual iteration can be reduced simply carrying out an adequate translation of specifications.

The use of successive approximations to the time parameter searched, applying any inverse interpolation method [14], specific for each type of requirement, will provide a tool for reducing manual iteration in the design process.

Specific techniques for specifications translation developed will be based on the "Newton's method by ascending and descending differences of $5^{\text {th }}$ order". This method was chosen over other traditional ones for interpolation, such as the Lagrange's polynomial, because of its practical advantages [11]:

- It requires less arithmetic operations. 
- No need to restart the computer, if you want to add or remove a set point that is used to construct the final polynomial.

In this sense, the first thing to consider is the type of function and, secondly, the temporal response characteristic to interpolate. The function is directly related to the type of specification to be treated.

- There are the specifications based on responses to step inputs tracking:

- Upper limit tracking, an input reference with maximum value " $r_{\max }$ " will be tracked by an output which must not exceed the amplitude " $y_{\max }$ " [18]. See Figure 1, case (a)

- Lower limit tracking, an output signal will track a step input in an underdamped way, not exceeding a maximum specific values of overshoot, rise time, settling time and settlement channel [20]. See example in Figure 2.

- Also, there are specifications associated with responses typed underdamped pulse, induced by an input disturbance, such as:

- Generic sensitivity, a disturbance "In" produces an output response pulse, not exceeding maximum specific amplitude "Out" and settling on after a specific maximum time $T$ [5]. See in Figure 1, case (b).

- Specific sensitivity, an input disturbance generates a pulse output between two specific values " $\left[ \pm\right.$ Out, \pm Out $\left._{\max }\right]$ ", but for a limited time $T$, settling into a channel with amplitude \pm Out [7]. See example in Figure 4.

(a) Input Data: $y_{\max }, r_{\max }, \mathrm{dev}$

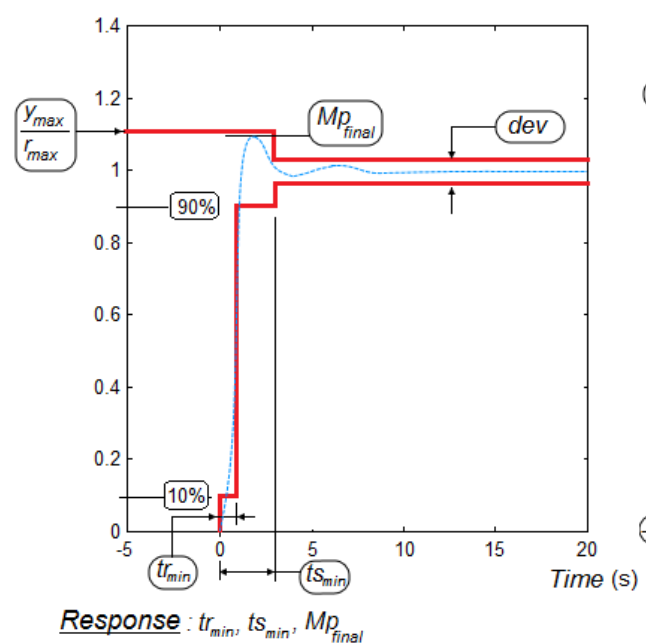

(b) Input Data : Out, In, T, dev

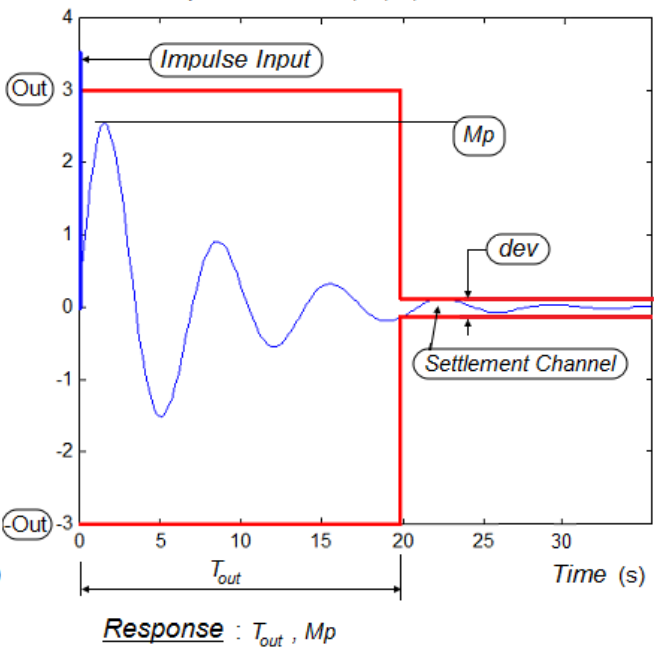

\section{Figure 1. Specification Examples: (a) Upper Frequency Tracking Limit (Minimum Times and Maximum Overshoot), (b) Generic Sensitivity (Upper Frequency Limit)}

The main objective of this work is to associate each time specification indicated with a transfer function, representative of a second order underdamped system with parameters $\omega_{n}$ and $\zeta$, as an approximation in the frequency domain. Why use a second order approximation and not any other order? With this type of approach the process can be automatic. In [15] is also developed an approach to the envelope of lower and upper limits for tracking specifications based on higher order systems, but requires manual implementation procedure; compared to the automatic second order method, it is concluded that are not better the results with the higher-order system.

In the second order approximation, the way to relate the specification response (time domain) with the equivalent parameters $\omega_{n}$ and $\zeta$ (frequency domain) is through the function that formally describes the temporal response generated by the specification. That is, using the descriptive function of the unitary step signal or the 
unitary pulse signal. Thus, it is used as a time dependent function any of the following:

$$
\begin{aligned}
& f_{\text {step }}(t)=1-\frac{e^{-\zeta \omega_{n} t}}{\sqrt{1-\zeta^{2}}} \operatorname{sen}\left(\omega_{n} \sqrt{1-\zeta^{2}} t+a \cos \zeta\right) \\
& f_{\text {pulse }}(t)=\frac{\omega_{n}}{\sqrt{1-\zeta^{2}}} e^{-\zeta \omega_{n} t} \operatorname{sen}\left(\omega_{n} \sqrt{1-\zeta^{2}} t\right)
\end{aligned}
$$

Observe how in the functions (1) and (2) is implicit the temporal relationship to the frequency domain, through the parameters $\omega_{n}$ and $\zeta$.Therefore, they can be used as interpolation functions of times $\left(t_{r}\right.$ and/or $\left.t_{s}\right)$ for different values of $\omega_{n}$ and $\zeta$. In practice, the response characteristics to interpolate for each of the functions indicated will be,

- The rise time and settling time of the step function (1), when working with tracking specifications of upper or lower limit.

- The rise time and/or the settling time of the pulse function (2), when working with generic or specific sensitivity specifications.

On the other hand, it can be argued that there are powerful tools such as Matlab functions that solve transcendental equations as proposed above. For example, with "solve" and "fsolve" really is so. In particular, "solve" searches analytical solutions (exact), while "fsolve" solves nonlinear equations by Gauss-Newton's method of least squares, using a mix of quadratic and cubic potential functions approximation. However, in the latter case, there is not an interpolation procedure, but an alternative method of nonlinear parametric adjustment [19]; i.e. "fsolve" can solve transcendental equations as proposed but, using an adjustment method, approximates to the function data, not to the function itself, as interpolation methods do; also, the approach is in one strict way, while the technique of differences that we use here, decides at each point if is more interested the progressive case (ascending) or the regressive one (descending), optimizing then the approach to each point and, obtaining a final function with minimal error respect to the initial desired. Moreover, it is important to note that in the differences method proposed is selected a specific range of interpolation for each equation to be treated, in each particular situation, while "solve" and "fsolve" use generic criteria applicable to any function. This makes these functions are not able to find the desired times in some situations, particularly the settlement one, on the step/pulse function considered; specifically, for $t_{s}$ you need to search by intervals (between peak to valley or peak to peak) and, "solve/fsolve" work with the whole function directly. On the other hand, "solve" provides solutions in the complex plane (by requiring an exact solution), which in the case of step/pulse functions don't interest; searching temporary response characteristics by interpolation are only valid real positive solutions, which "solve" does not give in many cases.

In conclusion, we will develop specifically independent technical for interpolation of temporal response characteristics on the step function and the pulse function, adapted and optimized to the type of specification that is intended to translate. In addition, a comparison of results will take place between interpolation techniques presented and resolution of the same problems with functions "solve" and "fsolve". 


\section{Inverse Interpolation Technical on the Step Function}

It will be used a technique of inverse interpolation on the step function (1), for the translation of tracking specifications to the frequency domain, for both the upper limit, as the lower. We will try to find the set of possible pairs $\left(\omega_{n}, \zeta\right)$ associated with the underdamped second order functions that fulfill all temporary requirements for tracking required.

The technique described in this section is an outline of the inverse interpolation technique on the step function, which can be found developed with more detail in [14] and [15].

It is used automatic iteration process, within the range of subcritical damping and limited by the maximum overshoot imposed (i.e., $1>\zeta>\zeta_{\min }$, with $\zeta_{\min }$ corresponding to $M p$ ), so that for each damping coefficient is sought:

1. On one hand, the rise time $t_{r}$ specified, associated to the pair $\left(\omega_{n r}, \zeta\right)$. To do this, use the following relationship, demonstrated in [15]:

$$
\frac{t r_{1}}{t_{r}}=\omega_{n r}
$$

That is, given the initial temporary specification $t_{r}$ and, calculated by interpolation $t r_{1}$ associated to the pair $\left(\omega_{n}=1, \zeta\right)$, is obtained from (3) the value $\omega_{n r}$, related to $t_{r}$ for the coefficient $\zeta$.

2. On the other hand, it is sought the settlement time $t_{s}$ required too, from which the response is adapted to a particular settlement channel and, which corresponds another pair $\left(\omega_{n s}, \zeta\right)$. To do this, use the next relationship obtained from [15].

$$
\frac{t s_{1}}{t_{s}}=\omega_{n s}
$$

So, with the temporary specification $t_{s}$, is calculated by interpolation $t s_{1}$ associated to the pair $\left(\omega_{n}=1, \zeta\right)$, and then the parameter $\omega_{n s}$ can get clearing in (4), related to $t_{s}$ for the damping coefficient $\zeta$.

3. Choose as possible valid pair $\left(\omega_{n}, \zeta\right)$, the one which represents the most restrictive condition for temporary joint condition $\left(t_{r}, t_{s}\right)$ : for the lower frequency limit specification, $\omega_{n}$ will be the greater value between $\omega_{n r}$ and $\omega_{n s}$; for the upper frequency limit specification, $\omega_{n}$ will be the lower value between both.

4. Determine times $t r_{\text {final }}$ and $t s_{\text {final }}$ associated with each possible pair $\left(\omega_{n}, \zeta\right)$ found, again using on (1) inverse interpolation; if both $\operatorname{tr}_{\text {final }}$ and $t s_{\text {final }}$ fulfill the initial requirements given for $t_{r}$ and $t_{s}$, the pair $\left(\omega_{n}, \zeta\right)$ associated will serve to generate a second order transfer function, possible solution in frequency.

Note that, in any case of interpolation, the natural frequency is a parameter given: initially $\omega_{n}=1$ or $\omega_{n}$ found later to verify possible validity of the pair $\left(\omega_{n}, \zeta\right)$.

Obtaining the equations (3) and (4) is detailed in [15], and the sense of the steps outlined above, when attempting to translate temporary specifications associated with step-like responses.

\subsection{Inverse Interpolation of the Rise Time $t_{r}\left(\omega_{n}, \zeta\right)$ on the Step Function}

The rise time $t_{r}$ of an underdamped step response is common to define between 10 and $90 \%, 5$ to $95 \%$ or 0 to $100 \%$, of its final value [21]. Here, we will apply the first interval (10 to $90 \%$ ), because it is more complex, although for underdamped systems is more used the interval 0 to $100 \%$. Therefore, using Alc as range 
percentage parameter of final steady value, $t_{r}$ is defined associated to the pair $\left(\omega_{n}, \zeta\right)$ as,

$$
t_{r}\left(\omega_{n}, \zeta\right)=t r_{90}\left(\omega_{n}, \zeta, A l c=90 \%\right)-t r_{10}\left(\omega_{n}, \zeta, A l c=10 \%\right)
$$

Thus, in this case, the statement is reduced to develop a technique for parameter interpolation type-like $\operatorname{tr}_{A l c}\left(\omega_{n}, \zeta, A l c\right)$, on the second order step function $f(t)$ with subcritical damping. Considering that for any time $t$, the function $f(t)$ is described as in (1), then,

$$
f(t)=f_{\text {step }}(t), \quad \text { with } \quad 0<\zeta<1
$$

To $t r_{A l c}$, equation (6) takes the value,

$$
f\left(\operatorname{tr}_{A l c}\right)=\frac{A l c}{100}=1-\frac{e^{-\zeta \omega_{n} t_{A l c}}}{\sqrt{1-\zeta^{2}}} \operatorname{sen}\left(\omega_{n} \sqrt{1-\zeta^{2}} t r_{A l c}+a \cos \zeta\right)
$$

If used for interpolation of $f(t)$ the Newton's method by differences, accurately of 5 th order, generic formulas to be applied are as follows [1]:

- In the case of "Descending Differences":

$$
f\left(t_{0}+\theta h\right)=f\left(t_{0}\right)+\delta_{d}{ }^{{ }^{\circ}} \theta+\frac{\delta_{d}^{2^{\circ}}}{2} \theta(\theta-1)+\frac{\delta_{d}^{3^{\circ}}}{3 !} \theta(\theta-1)(\theta-2)+\frac{\delta_{d}^{4^{\circ}}}{4 !} \theta(\theta-1)(\theta-2)(\theta-3)+\frac{\delta_{d}^{5^{\circ}}}{5 !} \theta(\theta-1)(\theta-2)(\theta-3)(\theta-4)
$$

- In the case of "Ascending Differences":

$$
f\left(t_{0}+\theta h\right)=f\left(t_{0}\right)+\delta_{a}^{1^{\circ}} \theta+\frac{\delta_{a}^{2^{\circ}}}{2} \theta(\theta+1)+\frac{\delta_{a}^{3^{\circ}}}{3 !} \theta(\theta+1)(\theta+2)+\frac{\delta_{a}^{4^{\circ}}}{4 !} \theta(\theta+1)(\theta+2)(\theta+3)+\frac{\delta_{a}^{5^{\circ}}}{5 !} \theta(\theta+1)(\theta+2)(\theta+3)(\theta+4)
$$

Where $h$ is the time interval chosen for differences $\delta^{i}$ of order $i\left(1^{\circ}, 2^{\circ}, ..\right)$. The general procedure consists of calculating the parameter $\theta$ per iteration. To do this, $\theta=F(\theta)$ is cleared and assigned an initial value $F\left(\theta_{0}\right)=\theta_{1}$.

If $\theta_{1}$ is appropriate, the process is terminated. Otherwise, continue assigning values $F\left(\theta_{1}\right)=\theta_{2}, F\left(\theta_{2}\right)=\theta_{3}, . .$, until a result is obtained such that, $\left|\theta_{i^{-}} \theta_{i-1}\right|<\left|\theta_{i-1}-\theta_{i-2}\right|$, with $i=1,2$, ..

Then, clearing $\theta$ in equation (8) or (9); for example, in the descending differences (8) and, substituting, it has got,

$$
\theta=\frac{f\left(t_{0}+\theta h\right)-f\left(t_{0}\right)}{\delta_{d}^{1^{\circ}}}-\frac{\delta_{d}^{2^{\circ}}}{2 \delta_{d}^{1^{\theta}}} \theta(\theta-1)+\frac{\delta_{d}^{3^{\circ}}}{3 ! \delta_{d}^{1^{\circ}}} \theta(\theta-1)(\theta-2)+\frac{\delta_{d}^{4^{\circ}}}{4 ! \delta_{d}^{1^{10}}} \theta(\theta-1)(\theta-2)(\theta-3)+\frac{\delta_{d}^{5^{\circ}}}{5 ! \delta_{d}^{1^{10}}} \theta(\theta-1)(\theta-2)(\theta-3)(\theta-4)
$$

To start the iteration is chosen,

$\theta_{0}=\frac{f\left(t_{0}+\theta h\right)-f\left(t_{0}\right)}{\delta_{d}^{1^{\circ}}}$

For generic function (6) in question, the particular sequence of steps to be taken would be:

1. Define the time interval $h$. Since the maximum overshoot $M p$ is reached in time $t_{M p}$, a valid practical value of interval is $1 / 10$ of it. That is to say, 
$t_{M p}=\frac{\pi}{\omega_{n} \sqrt{1-\zeta^{2}}} \Rightarrow h=\frac{\pi}{10 \omega_{n} \sqrt{1-\zeta^{2}}}$

And as discrete times using the following,

$t_{i}=\left\{t_{1}=0 ; t_{2}=t_{1}+h ; \ldots ; t_{n}=t_{n-1}+h\right\}$, with $i=1, \ldots, n$

Later, we will see that the choice of interval $h$ of $10 \% t_{M p}$ is not random. For the moment, consider that to cover with intervals $h$ the time between $0\left(t_{l}\right)$ and $t_{M p}$ $\left(t_{11}\right)$ is needed 11 time instants, ie, $n=11$.

2. Replace the values of earlier times (13) in the function $f(t)$ (6), taking into account the known value of $\omega_{n}$

3. Obtain the differences $\delta$ of $1^{\text {st }}, 2^{\text {nd }}, 3^{\text {rd }}, 4^{\text {th }}$ and $5^{\text {th }}$ order, from the relationships expressed in a table of differences, such as the one built in Table 1. Note how to obtain a differences table of $5^{\text {th }}$ order is necessary to sample 11 time instants. This is the reason to be for the chosen value $h$ in (12). The user can increase the accuracy of interpolation using interpolation intervals $h$ smaller, for example, $1 / 20$ or $1 / 30$ times the $t_{M p}$

4. Find the $t_{0}$ parameter to define the initial parameter $\theta_{0}(11)$, choosing the most appropriate differences procedure (ascending or descending), from the following criteria (for example in Table 1 above, with 11 time instants sampled),

$$
\begin{aligned}
& \text { (If } f\left(t_{i}\right)>\frac{A l c}{100} \text {, with } i=1,2, . ., 6 \text {, Then use, } \\
& \text { ○ Descending Differences and, } f\left(t_{0}\right)=f\left(t_{i-1}\right)<f(t) \text {, with } t_{i}<t<t_{i+1} \\
& \text { ○ Ascending Differences and, } f\left(t_{0}\right)=f\left(t_{i}\right)>f(t) \text {, with } t_{i-1} \leq t<t_{i}
\end{aligned}
$$

\begin{tabular}{|c|c|c|c|c|c|}
\hline$f(t)$ & $1^{\text {st }}$ Order & $2^{\text {nd }}$ Order & $3^{\text {rd }}$ Order & $4^{\text {th }}$ Order & $5^{\text {th }}$ Order \\
\hline$f\left(t_{1}\right)$ & $\delta_{11}=f\left(t_{2}\right)-f\left(t_{1}\right)$ & $\delta_{21}=\delta_{12}-\delta_{11}$ & $\delta_{31}=\delta_{22}-\delta_{21}$ & $\delta_{41}=\delta_{32}-\delta_{31}$ & $\delta_{51}=\delta_{42}-\delta_{41}$ \\
\hline$f\left(t_{2}\right)$ & $\delta_{12}=f\left(t_{3}\right)-f\left(t_{2}\right)$ & $\delta_{22}=\delta_{13}-\delta_{12}$ & $\delta_{32}=\delta_{23}-\delta_{22}$ & $\delta_{42}=\delta_{33}-\delta_{32}$ & $\delta_{52}=\delta_{43}-\delta_{42}$ \\
\hline$f\left(t_{3}\right)$ & $\delta_{13}=f\left(t_{4}\right)-f\left(t_{3}\right)$ & $\delta_{23}=\delta_{14^{-}} \delta_{13}$ & $\delta_{33}=\delta_{24}-\delta_{23}$ & $\delta_{43}=\delta_{34}-\delta_{33}$ & $\delta_{53}=\delta_{44}-\delta_{43}$ \\
\hline$f\left(t_{4}\right)$ & $\delta_{14}=f\left(t_{5}\right)-f\left(t_{4}\right)$ & $\delta_{24}=\delta_{15^{-}} \delta_{14}$ & $\delta_{34}=\delta_{25}-\delta_{24}$ & $\delta_{44}=\delta_{35}-\delta_{34}$ & $\delta_{54}=\delta_{45^{-}} \delta_{44}$ \\
\hline$f\left(t_{5}\right)$ & $\delta_{15}=f\left(t_{6}\right)-f\left(t_{5}\right)$ & $\delta_{25}=\delta_{16^{-}} \delta_{15}$ & $\delta_{35}=\delta_{26}-\delta_{25}$ & $\delta_{45}=\delta_{36^{-}} \delta_{35}$ & $\delta_{55}=\delta_{46}-\delta_{45}$ \\
\hline$f\left(t_{6}\right)$ & $\delta_{16}=f\left(t_{7}\right)-f\left(t_{6}\right)$ & $\delta_{26}=\delta_{17}-\delta_{16}$ & $\delta_{36}=\delta_{27}-\delta_{26}$ & $\delta_{46}=\delta_{37}-\delta_{36}$ & $\delta_{56}=\delta_{47}-\delta_{46}$ \\
\hline$f\left(t_{7}\right)$ & $\delta_{17}=f\left(t_{8}\right)-f\left(t_{7}\right)$ & $\delta_{27}=\delta_{18^{-}} \delta_{17}$ & $\delta_{37}=\delta_{28^{-}} \delta_{27}$ & $\delta_{47}=\delta_{38}-\delta_{37}$ & \\
\hline$f\left(t_{8}\right)$ & $\delta_{18}=f\left(t_{9}\right)-f\left(t_{8}\right)$ & $\delta_{28}=\delta_{19^{-}} \delta_{18}$ & $\delta_{38}=\delta_{29}-\delta_{28}$ & & \\
\hline$f\left(t_{9}\right)$ & $\delta_{19}=f\left(t_{10}\right)-f\left(t_{9}\right)$ & $\delta_{29}=\delta_{110^{-}} \delta_{19}$ & & & \\
\hline$f\left(t_{10}\right)$ & $\delta_{110}=f\left(t_{11}\right)-f\left(t_{10}\right)$ & & & & \\
\hline$f\left(t_{11}\right)$ & & & & & \\
\hline
\end{tabular}

That is, the nearest "point" with higher value than desired.

Being $t$ the time to find, in this case defined as $t r_{A l c}$

Table 1. Differences $\delta$ for functions $f(t)$ of $1^{\text {st }}, 2^{\text {nd }}, 3^{\text {rd }}, 4^{\text {th }}$ and $5^{\text {th }}$ order

Descending differences: Start at the last value $f(t 11)$

Ascending differences: Start at the first value $f(t 1)$

5. Calculate initial $\theta_{0}$, using the expressions, 
$f\left(t_{0}+\theta h\right)=\frac{A l c}{100} \Rightarrow \theta_{0}=\frac{\frac{A l c}{100}-f\left(t_{0}\right)}{\delta_{1 i}}$

6. Calculate the values $\theta_{i}(i=1,2, .$.$) and the error in each iteration: it is used to$ control the correct or not loop output $\left(\right.$ Error $\left._{i-1}=\left|\theta_{i-1}-\theta_{i}\right|\right)$, besides the accuracy of the approximation.

If descending differences, use:

$$
\begin{aligned}
& \left\{\begin{array}{l}
\theta_{1}=\theta_{0}-\frac{\delta_{d}^{2^{\circ}}}{2 \delta_{d}^{\mathrm{l}^{0}}} \theta_{0}\left(\theta_{0}-1\right)-\frac{\delta_{d}^{3^{\circ}}}{3 ! \delta_{d}^{\mathrm{I}^{0}}} \theta_{0}\left(\theta_{0}-1\right)\left(\theta_{0}-2\right)-\frac{\delta_{d}^{4^{0}}}{4 ! \delta_{d}^{\mathrm{l}^{0}}} \theta_{0}\left(\theta_{0}-1\right)\left(\theta_{0}-2\right)\left(\theta_{0}-3\right)-\frac{\delta_{d}^{5^{\circ}}}{5 ! \delta_{d}^{1^{0}}} \theta_{0}\left(\theta_{0}-1\right)\left(\theta_{0}-2\right)\left(\theta_{0}-3\right)\left(\theta_{0}-4\right) \\
\text { Error }_{0}=\left|\theta_{0}-\theta_{1}\right|
\end{array}\right. \\
& \left\{\begin{array}{l}
\theta_{2}=\theta_{1}-\frac{\delta_{d}^{2^{\circ}}}{2 \delta_{d}^{1^{10}}} \theta_{1}\left(\theta_{1}-1\right)-\frac{\delta_{d}^{3^{\circ}}}{3 ! \delta_{d}^{0^{10}}} \theta_{1}\left(\theta_{1}-1\right)\left(\theta_{1}-2\right)-\frac{\delta_{d}^{4^{\circ}}}{4 ! \delta_{d}^{0^{10}}} \theta_{1}\left(\theta_{1}-1\right)\left(\theta_{1}-2\right)\left(\theta_{1}-3\right)-\frac{\delta_{d}^{5^{\circ}}}{5 ! !_{d}^{1^{0}}} \theta_{1}\left(\theta_{1}-1\right)\left(\theta_{1}-2\right)\left(\theta_{1}-3\right)\left(\theta_{1}-4\right) \\
\text { Error }_{1}=\left|\theta_{1}-\theta_{2}\right|
\end{array}\right.
\end{aligned}
$$

Apply the following process:

$\left\{\begin{array}{l}\text { If Error } l<E p s, \text { then } \theta=\theta_{2} \text { and } t=t_{0}+\theta h \\ \text { If Error } 1 \geq E p s \text {, then continue iteration. }\end{array}\right.$

Note 1: The accuracy obtained depends on the 'Eps' user-defined parameter, that tells you the maximum error allowed in the iteration of applied research.

If ascending differences, use:

$$
\begin{aligned}
& \left\{\begin{array}{l}
\theta_{1}=\theta_{0}-\frac{\delta_{a}^{2^{\circ}}}{2 \delta_{a}^{1^{\circ}}} \theta_{0}\left(\theta_{0}+1\right)-\frac{\delta_{a}^{3^{\circ}}}{3 ! \delta_{a}^{1^{0}}} \theta_{0}\left(\theta_{0}+1\right)\left(\theta_{0}+2\right)-\frac{\delta_{a}^{4^{\circ}}}{4 ! \delta_{a}^{1^{0}}} \theta_{0}\left(\theta_{0}+1\right)\left(\theta_{0}+2\right)\left(\theta_{0}+3\right)-\frac{\delta_{a}^{5^{\circ}}}{5 ! \delta_{a}^{1^{\circ}}} \theta_{0}\left(\theta_{0}+1\right)\left(\theta_{0}+2\right)\left(\theta_{0}+3\right)\left(\theta_{0}+4\right) \\
\text { Error }_{0}=\left|\theta_{0}-\theta_{1}\right|
\end{array}\right. \\
& \left\{\begin{array}{l}
\theta_{2}=\theta_{1}-\frac{\delta_{a}^{2^{\circ}}}{2 \delta_{a}^{1^{10}}} \theta_{1}\left(\theta_{1}+1\right)-\frac{\delta_{a}^{3^{\circ}}}{3 ! \delta_{a}^{1^{0}}} \theta_{1}\left(\theta_{1}+1\right)\left(\theta_{1}+2\right)-\frac{\delta_{a}^{4^{\circ}}}{4 ! \delta_{a}^{1^{10}}} \theta_{1}\left(\theta_{1}+1\right)\left(\theta_{1}+2\right)\left(\theta_{1}+3\right)-\frac{\delta_{a}^{5^{\circ}}}{5 ! \delta_{a}^{1^{10}}} \theta_{1}\left(\theta_{1}+1\right)\left(\theta_{1}+2\right)\left(\theta_{1}+3\right)\left(\theta_{1}+4\right) \\
\text { Error }_{1}=\left|\theta_{1}-\theta_{2}\right|
\end{array}\right.
\end{aligned}
$$

Apply the following process:

$\left\{\begin{array}{l}\text { If Error } l<E p s, \text { then } \theta=\theta_{2} \text { and } t=t_{0}+\theta h \\ \text { If Error } l \geq E p s \text {, then continue iteration. }\end{array}\right.$

Note 2: For each $\theta_{i}(i=1,2, .$.$) , then \delta^{i^{\circ}}=\delta_{I i}, \delta^{2^{\circ}}=\delta_{2 i}, \ldots$

\subsection{Inverse Interpolation of the Time $t_{s}\left(\omega_{n}, \zeta, d e v\right)$ on the Step Function}

The search procedure for settling time $t_{s}$ required is, in principle, the same as described for the previous rise time $t_{r}$, except for the following special considerations:

- With the parameter dev, it is defined the settlement channel with values in unitary percentage in the range given by, 
$1 \pm \frac{\text { desv }}{100}$

- To obtain the settling time, it is going to observe the behavior of each signal semicycles at the zero-maximum_peak interval, for the first and, maximum_peak-minimum_peak, for the others:

- If the first maximum peak is within the settlement channel, the settling time will be in the first semicycle, in the ascending interval (rise), before the peak considered.

- If not, when a full semicycle (maximum_peak-minimum_peak) is within the settlement channel, time sought will be within the previous semicycle; otherwise, the settling time will be the time in reaching firstly the settlement channel plus the time sum of every semicycles $n \pi / \omega_{n} \sqrt{1-\zeta^{2}}$, until the signal is within the channel.

The inverse interpolation of $t_{s}$ is performed between 0 and $t_{l}$ (first semicycle), $t_{l}$ and $t_{2}$ (second semicycle) and, so on until you find the appropriate value (Figure 3 ).

It is true that for the determination of establishment time you can use the envelope of the function described in (1), without losing too much precision and, therefore, no numerical method would be necessary to find these parameters. However, the interpolation method proposed has been developed to search for rise times, impossible to determine accurately through the envelope said and, its use takes advantage of determining with more accurate $t_{s}$ or combinations type-like $t_{s}-t_{r}$, such as those that will be proposed in section 4 on the pulse function.

\subsection{Example application of Inverse Interpolation on the Step Function}

In the example of Figure 2, it has been used the application TD2WD.M, as indicated in Section 5, for obtaining the lower frequency limit in tracking. Resolved (6) applying different procedures,

1. The inverse interpolation technique described in Section 3.

2. The function 'solve' on independent equations with one unknown $\left(\omega_{n}\right.$ and $\zeta$ known), combined with the procedure associated with equations (3) and (4).

3. The 'fsolve' function with one unknown equations, combined with the procedure associated with (3) and (4).

4. The 'solve' function on a system of four equations with unknowns, $\operatorname{tr}_{10}\left(\omega_{n}, \zeta, A l c=10 \%\right), \operatorname{tr}_{90}\left(\omega_{n}, \zeta, A l c=90 \%\right), \omega_{n}$ and $t_{s}\left(\omega_{n}, \zeta\right)$. The time $t_{s}$, in this case, is described from the equation of the envelope on the step function, i.e. with,

$$
f\left(t_{s}\right)=1-\frac{e^{-\zeta \omega_{n} t_{s}}}{\sqrt{1-\zeta^{2}}}
$$

5. The 'fsolve' function on a system of four equations with the unknowns indicated in paragraph 4 above.

A summary of the results achieved is described in comparison between procedures of Table 2, where it is given the deviation obtained with each, as to the rise time $\left(f\left(t_{r}\right)-f(t)\right)$ and the settlement time $\left(f\left(t_{s}\right)-f(t)\right)$.

a) Temporary specifications required were: $30 \%$ overshoot; maximum rise and settlement times of $5 \mathrm{~s}$ and $20 \mathrm{~s}$, respectively, on a $\pm 4 \%$ settlement channel. 
Table 2. Results and Deviations Obtained with the 5 Procedures Proposed, for the Example in Section 3.3

\begin{tabular}{|c|c|c|c|c|c|c|c|c|c|}
\hline \multirow{2}{*}{ Method } & \multicolumn{3}{|c|}{$f(t)=0.9$} & \multicolumn{3}{|c|}{$f(t)=0.1$} & \multicolumn{3}{|c|}{$f(t)=[0.96,1.04]$} \\
\hline & $t_{r}(s)$ & $f\left(t_{r}\right)$ & $f\left(t_{r}\right)-f(t)$ & $t_{r}(s)$ & $f\left(t_{r}\right)$ & $f\left(t_{r}\right)-f(t)$ & $t_{s}(s)$ & $f\left(t_{s}\right)$ & $f\left(t_{s}\right)-f(t)$ \\
\hline 1 & 6.4993 & 0.90116 & 0.00116 & 1.4993 & 0.0953 & -0.0047 & 17.0061 & 1.04 & 0 \\
\hline 2 & 10.0268 & 0.30058 & -0.5994 & 5.02678 & 0.1 & 0 & 10.2866 & 0.31177 & -0.6482 \\
\hline 3 & 3.6802 & 0.9 & 0 & 0.52787 & 0.1 & 0 & 20 & 1 & 0.04 \\
\hline 4 & No possible & - & - & No possible & - & - & No possible & - & - \\
\hline 5 & 5.8965 & 0.9 & 0 & 0.89645 & 0.1 & 0 & 20 & 1 & 0.04 \\
\hline
\end{tabular}

1 , Inverse interpolation by Newton's method of $5^{\text {th }}$ order

2, Function 'solve' on independent equations

3, Function 'fsolve' on independent equations

4, Function 'solve' on system of equations

5 , Function 'fsolve' on system of equations

b) Inverse interpolation response by Newton's method. Results shown in Figure 2a:

- Maximum time response characteristics:

- Final $M p$ of $19.83 \%$.

- Rise time calculated of $5 \mathrm{~s}$. Good deviations and perfect approach result to $t_{r}$ demanded.

- Settling time calculated of $17 \mathrm{~s}$. Exact approach, with no deviation.

(a) Inverse Interpolation

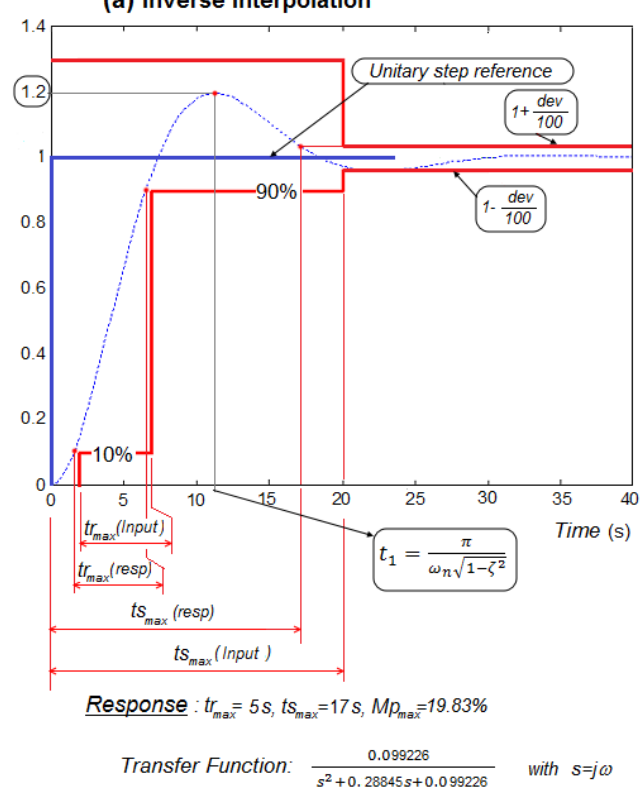

(b) 'Solve' with independent equations

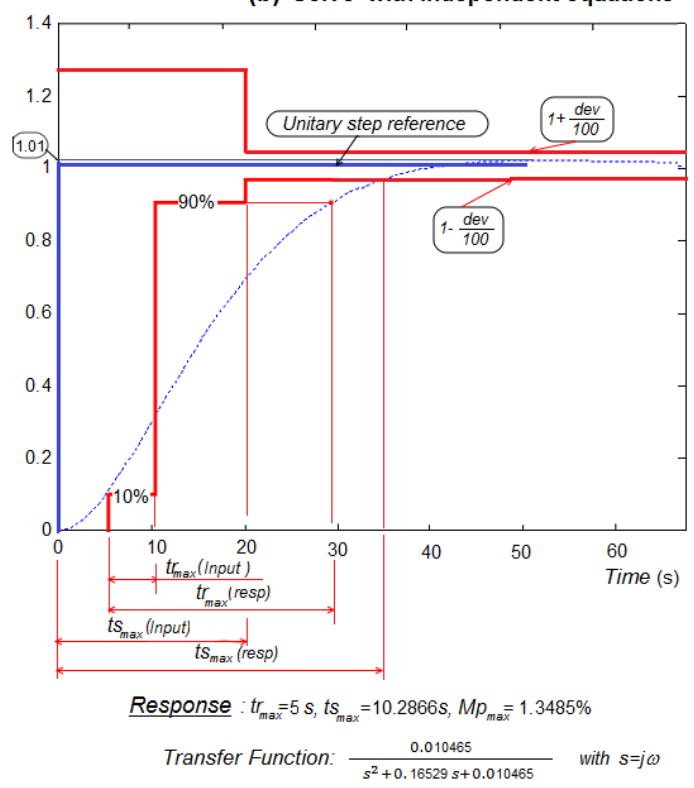

Figure 2. Example of Tracking Specification with Maximum Time Response Characteristics, (a) Approximated by Inverse Interpolation, (b) Approximated Applying 'Solve' with Independent Equations

c) The results obtained with the other four proposed comparative methods are:

- Response applying 'solve' with independent equations, shown in Figure 2b:

- Maximum time response characteristics:

- Final $M p$ of $1.35 \%$.

- Rise time calculated of $5 \mathrm{~s}$. But, because of the enormous deviation obtained for $f(t)=0.9(90 \%)$, the $t_{r}($ resp $)$ is almost $25 \mathrm{~s}$. 
- Settling time calculated of $10.29 \mathrm{~s}$. With the large deviation obtained for $f(t)=0.96, t_{s}($ resp $)$ is more than $35 \mathrm{~s}$.

- Response applying 'fsolve' with independent equations, shown in Figure 3a:

- Maximum time response characteristics:

- Final $M p$ of $0.0028 \%$.

- Rise time calculated of 3.15s. Perfect approach result to $t_{r}$ demanded.

- Settling time calculated of 20s. With the bad deviation obtained for $f(t)=0.96, t_{s}($ resp $)$ is about $5 \mathrm{~s}$ for $t_{s}$ (calculated) of $20 \mathrm{~s}$.

- Response using 'solve' with system of equations: For this example, there is no solution.

- Response applying 'fsolve' with equation system, shown in Figure 3b:

- Maximum time response characteristics:

- Final $M p$ of $0.11 \%$.

- Rise time calculated of $5 \mathrm{~s}$. Very good result that matches the required $t_{r}$.

- Settling time calculated of 20s. Bad results obtained for $f(t)=0.96$, so $t_{s}($ resp $)$ is about $8 \mathrm{~s}$ with $t_{s}$ (calculated) of $20 \mathrm{~s}$.

(a) 'Fsolve' with independent equations Input Data: $\operatorname{tr}_{\operatorname{ma}}=5 \mathrm{~s}, t \mathrm{~s}_{\max }=20 \mathrm{~s}, M p=30 \%$, dev $= \pm 4 \%$

(b) 'Fsolve' with system of equations
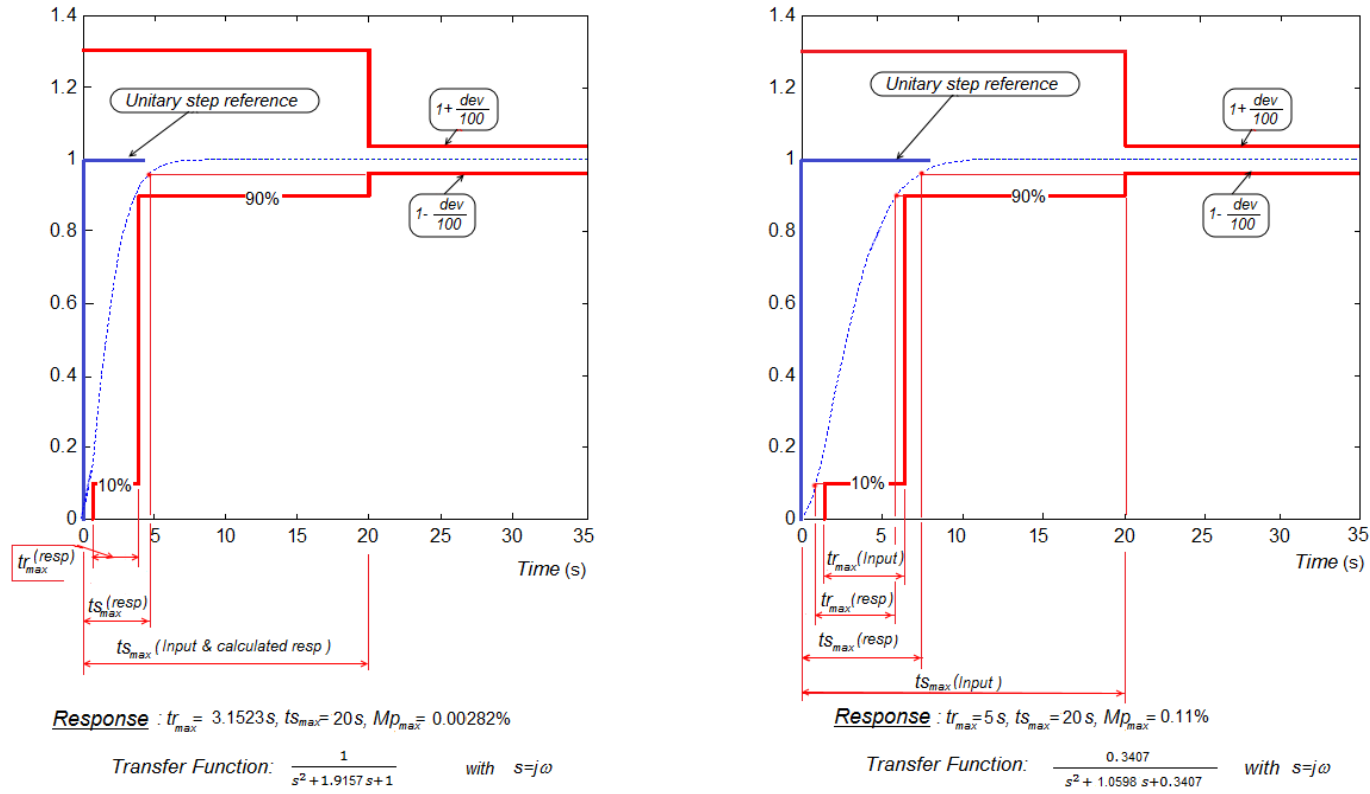

Figure 3. Example of Tracking Specification with Maximum Time Response Characteristics, (a) Approximated Applying 'Fsolve' with Independent Equations, (b) Approximated Applying 'Fsolve' with System of Equations

d) There is no any procedure comparable with the inverse interpolation proposed. Approaches for $t_{r}$ are good using 'fsolve' application, but no so to obtain $t_{s}$. In general, results are not practical, except for inverse interpolation.

\section{Inverse Interpolation Technical on the Pulse Function}

For translation of sensitivity specifications in the frequency domain, in both generic and specific, as defined in section 2, is applying an inverse interpolation technique on the pulse function (2). We will try to find the set of possible pairs $\left(\omega_{n}, \zeta\right)$ representing the second order functions, which fulfill all temporary requirements for regulation imposed. 
As is the case in section 3, the technique described here is an outline of the inverse interpolation technique on the pulse function, developed in detail in [14].

It is used an automatic iteration process, within the range of subcritical damping; i.e. $1>\zeta>0$, so that for each damping coefficient and depending on the sensitivity type considered, is sought:

1. For generic sensitivity, you only need to work with settling time $t_{s}$, which must not exceed the specified $T$, associated with the pair $\left(\omega_{n}, \zeta\right)$. To do this, use the relationship:

$$
\frac{t s_{1}}{t_{s}}=\omega_{n}
$$

That is, given $t_{s}=T$ as time specification and $t s_{1}$ associated with the pair $\left(\omega_{n}=1, \zeta\right)$, calculated by interpolation, it is obtained from (27) the parameter $\omega_{n}$, related to $t_{s}$ for the damping coefficient $\zeta$. The validity of the pair $\left(\omega_{n}, \zeta\right)$ is checked, applying again inverse interpolation on the function (2), to obtain $t s_{\text {final }}$, such that:

○ If $t s_{\text {final }}\left(\omega_{n}, \zeta\right) \leq T$ and maximum amplitude imposed is not exceeded, the pair $\left(\omega_{n}, \zeta\right)$ will be a possible second order solution in frequency.

2. For specific sensitivity, it is necessary to use inverse interpolation as on settling time $t_{s}$ as the rise time $t_{r}$, although associated in both cases at the same amplitude on the pulse function (2). To do this, use the relationship described by:

$$
\frac{t s_{1}-t r_{1}}{t_{s}}=\omega_{n}
$$

That is, given the time specification $t_{s}=T$, it is calculated by interpolation $\left(t s_{1}-t r_{l}\right)$, time difference associated with the same pair $\left(\omega_{n}=1, \zeta\right)$, clearing in (28) the parameter $\omega_{n}$, related with $t_{s}$ for the coefficient $\zeta$. Note that you have to find separate $t s_{1}$ and $t r_{l}$, so interpolation is used twice. The pair $\left(\omega_{n}, \zeta\right)$ obtained is checked by applying again inverse interpolation on the function (2) for getting $\left(t_{s}-t_{r}\right)_{\text {final }}$, such that:

$\circ$ If $\left(t_{s}-t_{r}\right)_{\text {final }}\left(\omega_{n}, \zeta\right) \leq T$ and maximum amplitude imposed is not exceeded, the pair $\left(\omega_{n}, \zeta\right)$ can be a possible solution.

Obtaining the equations (27) and (28) is detailed in [14] and, the sense of the steps outlined above, when trying to translate temporary specifications associated with pulse type responses. On the other hand, the approach is equivalent to that defined in section 3 , when working with step functions.

\subsection{Inverse Interpolation of the Rise Time $t_{r}\left(\omega_{n}, \zeta, A l c\right)$ on the Pulse Function}

It is about finding the rise time $t_{r}$ on pulse signal $f(t)$, when it takes the rising value "Alc" and is defined by the parameters $\omega_{n}$ and $\zeta$. The approach reduces to the technique development for parameter interpolation type-like $\operatorname{tr}_{A l c}\left(\omega_{n}, \zeta, A l c\right)$, on the second order pulse function $f(t)$ with subcritical damping.

The function $f(t)$ is defined for each time $t$, as in (2), that is:

$$
f(t)=f_{\text {pulse }}(t) \text {, with } 0<\zeta<1
$$

For $t r_{A l c}$, equation (29) takes the value,

$$
f\left(t r_{A l c}\right)=\frac{A l c}{100}=\frac{\omega_{n}}{\sqrt{1-\zeta^{2}}} e^{-\zeta \omega_{n} t_{A l c}} \operatorname{sen}\left(\omega_{n} \sqrt{1-\zeta^{2}} t r_{A l c}\right)
$$


Interpolation procedure by Newton's differences of $5^{\text {th }}$ order on $f\left(t_{A l c}\right)$ is the same as described in section 3.1, with the following exceptions:

1. Time interval $h$ of differences interpolation. Considering that the maximum peak value $f_{\max }$ is reached at the time instant $t_{\max }$ given by,

$$
t_{\max }=\frac{\arctan \left(\frac{\sqrt{1-\zeta^{2}}}{\zeta}\right)}{\omega_{n} \sqrt{1-\zeta^{2}}}
$$

A valid practical value of interval $h$ is $1 / 10$ of the time $t_{\max }$, for the same reason to define the interpolation interval used in section 3.1. That is,

$$
h=\frac{t_{\max }}{10}
$$

And use as discrete times those given in (13).

2. Substitute the values of previous times (13) in the interpolation function $f(t)$ (29), given the known value $\omega_{n}$.

From here, the interpolation technique follows the same steps as in section 3.1, i.e.:

3. Get the values $\delta$ of $1^{\text {st }}, 2^{\text {nd }}, 3^{\text {rd }}, 4^{\text {th }}$ and $5^{\text {th }}$ order, using Table 1 of differences.

4. Find the $t_{0}$ parameter, using the criteria described in section 3.1 from equations (14) and (15).

5. Calculate initial $\theta_{0}$ using (16) and the value $h$ in (32).

6. Calculate $\theta_{i}$ values $(i=1,2, .$.$) and the error in each iteration \left(\right.$ Error $\left._{i-1}=\left|\theta_{i-1}-\theta_{i}\right|\right)$, by a method as indicated between equations (17) and (24).

\subsection{Inverse Interpolation of the Time $t_{s}\left(\omega_{n}, \zeta, A s\right)$ on the Pulse Function}

The search procedure for settling time $t_{s}$ required, for the pulse function, is similar to that described for the rise time $t_{r}$ above, in section 4.1, except for the following special considerations (see Figure 4a):

$\circ$ The "As" parameter defines the settlement channel of pulse response with values in unitary percentage within the range given by,

$$
\pm \frac{A s}{100}
$$

- The settling time is obtained by observing the behavior of each signal semicycles in the range, peak (positive maximum) and valley (negative minimum) or valley-peak:

- If the first peak is within the settlement channel, then settling time $t_{s}$ is zero.

- If not, when a full semicycle (peak-valley or valley-peak) is located within the settlement channel, other than the first, the time $t_{s}$ will be associated with the previous semicycle; otherwise, the settling time will be this time, plus the sum of time of each semicycle $\frac{\pi}{\omega_{n} \sqrt{1-\zeta^{2}}}$, until the peak or valley of the function is within the channel.

- The temporal interpolation interval $h$ is provided between a peak or valley and the next zero function, temporarily located on $t_{p}$ and $t_{c}$, respectively. Where, 


$$
\begin{aligned}
& t_{p}=\frac{\arctan \left(\frac{\sqrt{1-\zeta^{2}}}{\zeta}\right)+n \pi}{\omega_{n} \sqrt{1-\zeta^{2}}} \\
& t_{c}=\frac{n \pi}{\omega_{n} \sqrt{1-\zeta^{2}}}
\end{aligned}
$$

Both with $n=0,1, \ldots$. Note that $t_{p}(n=0)=t_{\max }$

The inverse interpolation of $t_{s}$ within the range $h\left(t_{p}\right.$ to $\left.t_{c}\right)$ is between $t_{p}$ and $t_{1}, t_{l}$ and $t_{2}$, and so on, until completion in $t_{c}\left(t_{1}, t_{2}, . .\right.$, are discrete times given in (13)) .

\subsection{Example application of Inverse Interpolation on the Pulse Function}

In the examples of Figure 4 and Figure 5, it has been used the application ESENSIB.M, accessible from section 5, to obtain the lower frequency limit for specific sensitivity. Resolved (29) using the following procedures:

1. The inverse interpolation technique described in Section 4.

2. The function 'solve' on independent equations with one unknown ( $\omega_{n}$ and $\zeta$ known), combined with the procedure associated with equation (28).

3. The 'fsolve' function with one unknown equations, combined with the procedure associated with (28).

4. The 'solve' function on a system of three equations with unknowns, $\operatorname{tr}_{A l c}\left(\omega_{n}, \zeta, A l c=O u t\right), \omega_{n}$ and $t_{s}\left(\omega_{n}, \zeta\right)$. The time $t_{s}$, in this case, is described from the equation of the envelope on the pulse function, i.e. with,

$$
f(t s)=\frac{\omega_{n}}{\sqrt{1-\zeta^{2}}} e^{-\zeta \omega_{n} t s}
$$

5. The 'fsolve' function on a system of three equations with the unknowns indicated in the preceding paragraph.

Table 3: Results and Deviations Obtained with the 5 Proposed Procedures, for Example in Section 4.3

\begin{tabular}{|c|c|c|c|c|c|c|}
\hline \multirow{2}{*}{ Method } & \multicolumn{3}{|c|}{$\boldsymbol{f}(\boldsymbol{t})=\mathbf{0 . 2 5}$} & \multicolumn{3}{c|}{$\boldsymbol{f}(\boldsymbol{t})= \pm \mathbf{0 . 2 5}$} \\
\cline { 2 - 7 } & $\boldsymbol{t}_{\boldsymbol{r}}(\boldsymbol{s})$ & $\boldsymbol{f}\left(\boldsymbol{t}_{\boldsymbol{r}}\right)$ & $\boldsymbol{f}\left(\boldsymbol{t}_{\boldsymbol{r}}\right)-\boldsymbol{f}(\boldsymbol{t})$ & $\boldsymbol{t}_{\boldsymbol{s}}(\boldsymbol{s})$ & $\boldsymbol{f}\left(\boldsymbol{t}_{\mathbf{s}}\right)$ & $\boldsymbol{f}\left(\boldsymbol{t}_{\boldsymbol{s}}\right)-\boldsymbol{f}(\boldsymbol{t})$ \\
\hline 1 & 0.38 & 0.2498 & -0.0002 & 10.05 & 0.2501 & 0.0001 \\
\hline 2 & 0.45 & 0.25 & 0 & 6.31 & -0.3399 & -0.0899 \\
\hline 3 & $8.9 * 10^{-5}$ & $5.4 * 10^{-5}$ & -0.2500 & 5.91 & -0.3562 & -0.1062 \\
\hline 4 & 0.37 & 0.25 & 0 & 10.37 & 0.1613 & -0.0887 \\
\hline 5 & 0.54 & 0.2490 & -0.0010 & 10.54 & 0.2090 & -0.0411 \\
\hline
\end{tabular}

1 , Inverse interpolation by Newton's method of $5^{\text {th }}$ order

2, Function 'solve' on independent equations

3, Function 'fsolve' on independent equations

4, Function 'solve' on system of equations

5 , Function 'fsolve' on system of equations

Note: In Table 3 is being used $f(t)=O u t / I n$ 
A summary of the results achieved is described in comparison between procedures of Table 3, where it is given the deviation obtained with each, as to the rise time $\left(f\left(t_{r}\right)-f(t)\right)$ and the settlement time $\left(f\left(t_{s}\right)-f(t)\right)$.

a) Temporary specifications required are: amplitude of the pulse type response ranging between 1.5 and 4.35 , for no more than $10 \mathrm{~s}$. Therefore, the settlement channel is in the range \pm 1.5 .

b) Response by inverse interpolation, Newton's method. Results are shown in Figure 4a:

- Maximum time response characteristics:

- $f_{\max }=4.153$ which does not exceed the value imposed of 4.35

- Rise time calculated response 0.38s. For $f(t)=1.5$ (Out or Out/In=0.25), it is obtained the $t r_{\text {out }}$ indicated in the first semicycle, between 0 and $t_{c l}$.

- Settling Time calculated response, 10.05s. For $f(t)= \pm 1.5$ (settlement channel) the obtained $t s_{\text {out }}$ produces $T_{\text {out }}=t s_{\text {out }}-t r_{\text {out }}=9.67 \mathrm{~s} \leq 10 \mathrm{~s}$. The first semicycle full enter into settlement channel is number 4 .

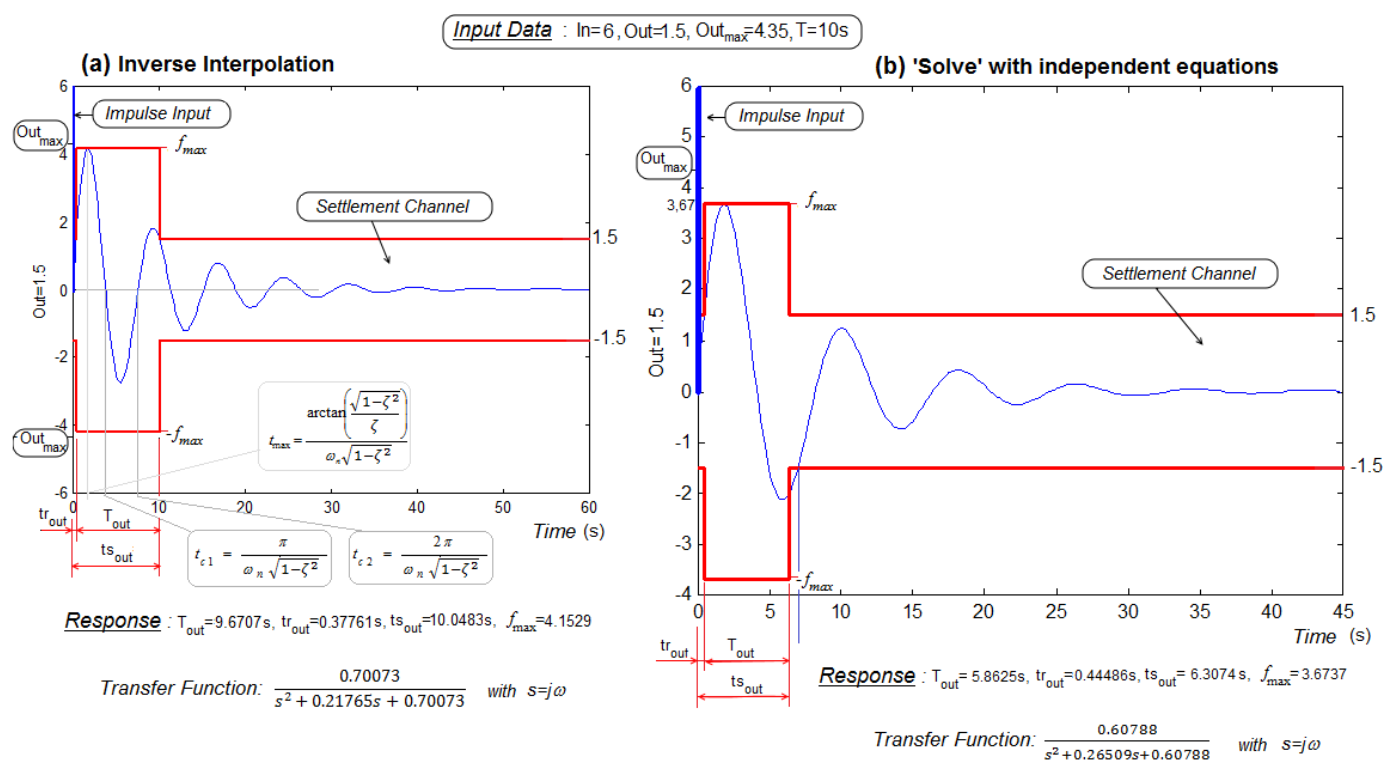

\section{Figure 4. Example of Sensitivity Specification with Characteristics of Maximum Response, (a) Approximated by Inverse Interpolation, (b) Applying 'Solve' with Independent Equations}

c) The results obtained with the other four proposed comparative methods are:

- Response applying 'solve' with independent equations, shown in Figure 4b:

- Maximum time response characteristics:

- $f_{\max }=3.674$ which does not exceed the value imposed of 4.35

- Rise time calculated response, $0.45 \mathrm{~s}$, for $f(t)=1.5$. No deviation.

- Settling time calculated response, 6.31s. For $f(t)= \pm 1.5$, it is obtained $T_{\text {out }}=t s_{\text {out }}-t r_{\text {out }}=5.86 \mathrm{~s} \leq 10 \mathrm{~s}$. High deviation, so the actual $T_{\text {out }}$ does not match.

- Response applying 'fsolve' with independent equations, shown in Figure 5a:

- Maximum time response characteristics:

- $f_{\max }=3.674$ which does not exceed the value imposed of 4.35

- Rise time calculated response, $9 * 10^{-5} \mathrm{~s}$, for $f(t)=1.5$. Very high deviation, so the $t r_{\text {out }}$ does not match the real.

- Settling Time calculated response, 5.91s. For $f(t)= \pm 1.5$, it is obtained $T_{\text {out }}=t s_{\text {out }}-t r_{\text {out }}=6.91 \mathrm{~s} \leq 10 \mathrm{~s}$. High deviation, so $T_{\text {out }}$ differs from the actual. 

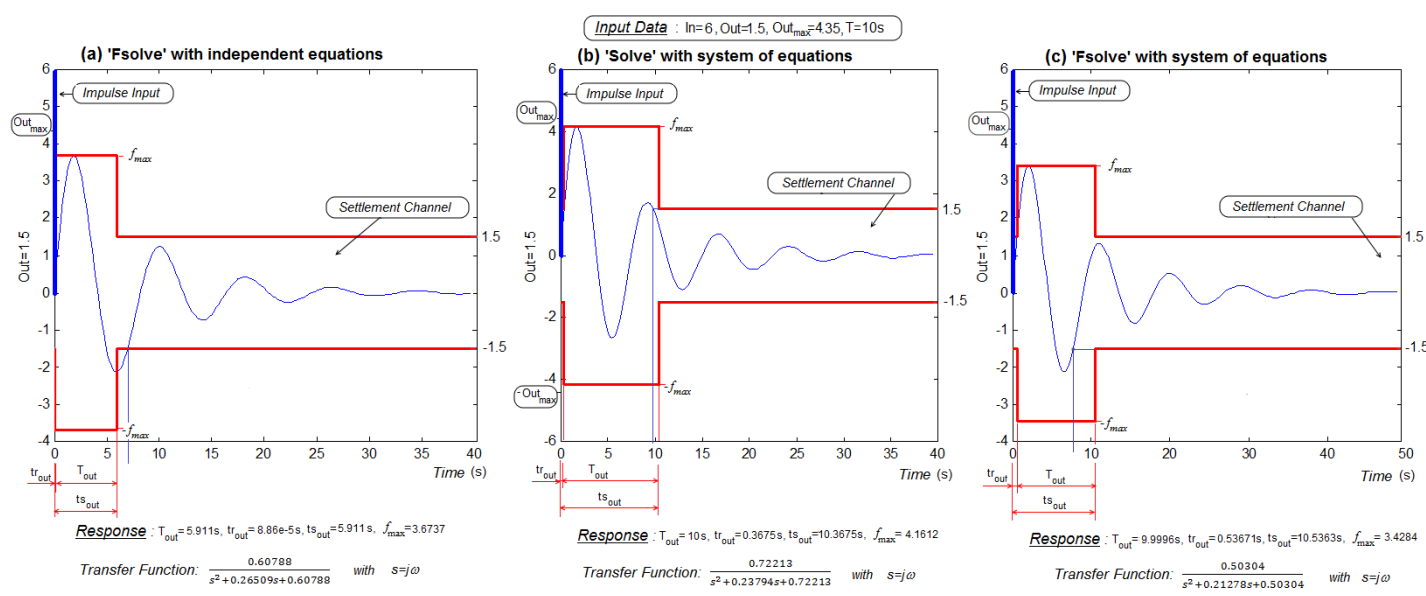

Figure 5. Example of Sensitivity Specification with Characteristics of Maximum Temporal Response, (a) Approximated Applying 'Fsolve' with Independent Equations, (b) Approximated Applying 'Solve' with System of Equations, (c) Approximated Applying 'Fsolve' with System of Equations

- Response using 'solve' with system of equations, shown in Figure 5b:

- Maximum time response characteristics:

- $f_{\max }=4.161$ which does not exceed the value imposed of 4.35

- Rise time calculated response, $0.3675 \mathrm{~s}$, for $f(t)=1.5$. No deviation.

- Settling Time response 10.3675s. For $f(t)= \pm 1.5$, it is obtained $T_{\text {out }}=t s_{\text {out }}-t r_{\text {out }}=10 \mathrm{~s} \leq 10 \mathrm{~s}$. High deviation, so the actual $T_{\text {out }}$ does not match.

- Response applying 'fsolve' with equation system, shown in Figure 5c:

- Maximum time response characteristics:

- $f_{\max }=3.428$ which does not exceed the value imposed of 4.35

- Rise time calculated response, $0.5367 \mathrm{~s}$, for $f(t)=1.5$. Correct deviation.

- Settling Time calculated response 10.5363s. For $f(t)= \pm 1.5$, it is obtained $T_{\text {out }}=t s_{\text {out }}-t r_{\text {out }}=9.9996 \mathrm{~s} \leq 10 \mathrm{~s}$. Middle deviation, so $T_{\text {out }}$ differs from the actual.

d) Noting that data obtained, maximum precision and final results are given by inverse interpolation, against the implementation of 'solve' and 'fsolve': compare deviations shown in Table 3 and graphically in Figure 4 and Figure 5.

\section{Application Development}

The author has developed in Matlab [2] applications to verify all examples given in this paper and also serve to translate the main temporary specifications to frequency domain already named.

The "Specifications Translation" folder contains the various techniques described for upper/lower limit tracking and generic/specific sensitivity, which can be downloaded from the address [22],

\section{Conclusions}

It has been discussed and presented sufficient arguments to justify the importance of having a methodology for temporary specifications translation to frequency domain with precise, automatic and specific character, for each type of requirement. To get it, a solution is to use successive approximations to the temporal response characteristic sought (rise time and/or settling time), applying an inverse interpolation method. 
Specific techniques for specifications translation presented will be based on the "Newton's method by ascending and descending differences of $5^{\text {th }}$ order", advantageous in practice against other traditional use for interpolation too, as can be Lagrange's polynomial.

Justified the need to use successive approximations by interpolation for specifications translation, next is to consider the type of function and characteristics of temporal response to interpolate. The function is directly related to the type of specification to be treated. Thus, for responses based on specifications for tracking step inputs, such as tracking lower limits and/or higher, the function is the step type. For sensitivity specifications, both general and specific, are based on underdamped pulse type responses. In any case, the function will be that descriptive of the step signal or pulse signal, with subcritical damping $(0<\zeta<1)$ and approximated by a second order system.

In this paper, it has been developed independent techniques, for interpolation of time response characteristics on the step function and the pulse function, adapted to the type of specification that is intended to translate.

The goodness of each is demonstrated by the proposed practical examples and comparative results raised, respect to the implementation of functions for direct solving equations, 'solve' and 'fsolve' in Matlab.

On the other hand, applications developed for each type of specification are offered with free access by the author, from the URL indicated in the previous section.

\section{References}

[1] J. F. Bonnans, J. C. Gilbert, C. Lemaréchal and C. A. Sagastizábal, "Numerical optimization: Theoretical and practical aspects", Universitext Berlin: Springer-Verlag,. DOI:10.1007/978-3-54035447-5. ISBN 3-540-35445-X. MR 2265882, (2006), pp. xiv+490.

[2] C. Borghesani, Y. Chait and O. Yaniv, "The QFT control desing Toolbox - for use with MATLAB", Terasoft Inc., (2003)

[3] J. D'Azzo and C. Houpis, "Linear control systems analysis and design: conventional and modern", New York, USA: McGraw-Hill, 4ªd, Cap18, (1995)

[4] M. A. Franchek and P. A. Herman, "Direct connection between time-domain performance and frequency-domain characteristics", International Journal of Robust and Nonlinear Control, 8(12), (1998), pp. 1021-1042.

[5] M. A. Franchek, P.A. Herman and O. D. I. Nwokah, "Robust nondiagonal controllers design for uncertain multivariable regulating systems", J. Dyn Syst-T ASME, 119, (1997), pp. 80-85.

[6] M. A. Franchek and O. D. I. Nwokah, "Robust multivariable control of distillation columns using nondiagonal controller matrix", IMECE, ASME Journal of Dynamic Systems and Control, 57-1, (1995), pp. 257-274.

[7] GARTEUR Action Group FM(AG08), "Robust flight control design challenge. Problem formulation and manual: the research civil aircraft model (RCAM)", GARTEUR/TP-088-3, (1997)

[8] M. Gil-Martínez, "Síntesis de controladores robustos mediante el análisis de la compatibilidad de especificaciones e incertidumbre", Ph.D. thesis, Universidad de la Rioja, Spain, (2008).

[9] I. M. Horowitz and M. Sidi, "Synthesis of feedback systems with large plant ignorance for prescribed time domain tolerances", International Journal of Control, 16, (1972), pp. 287-309.

[10] C. H. Houpis and S. J. Rasmussen, "Quantitative Feedback Theory: fundamentals and applications", Marcel Dekker Inc, New York, (1999).

[11] S. R. K. Iyengar, "Numerical methods and computation. Interpolation and approximation", Lecture $\mathrm{N}^{\mathrm{o}}$ 28, Dep. of Mathematics, Indian Institute of Technology, Delhi, (2008)

[12] S. Jayasuriya and M. A. Franchek, "Frequency domain design for maximal rejection of persistent bounded disturbances”, J. Dyn Syst-T ASME, 113(2), (1991), pp. 195-205.

[13] J. Joglar and J. Aranda, "QFT multivariable control for the longitudinal dynamics of an air vehicle", The $9^{\text {th }}$ International Conference on Electrical and Control Technologies, ECT2014, Lithuania, (2014), pp. 30-35.

[14] J. Joglar-Alcubilla, "Contribución al estudio de diseño de sistemas de control mediante QFT: aplicaciones al diseño de sistemas de control de vuelo y navegación”, Ph.D. thesis, ETSI Informática, UNED. Spain, (2015), March 10. 
[15] J. Joglar-Alcubilla, "Generic tracking specifications translation from time domain to frequency domain", ArXiv:1601.00346 [cs.SY], (2016), Jan16.

[16] J. Joglar Alcubilla, "Interpolación inversa de características de respuesta transitoria temporal a parámetros de la función de transferencia típica de segundo orden", Revista Iberoamericana de Automática e Informática Industrial, 14(1), (2017), pp. 44-55, DOI: 10.1007/978-3-540-35447-5

[17] K. R. Krishnan and A. Cruickshanks, "Frequency-domain design of feedback systems for specified insensitivity of time-domain response to parameter variation", International Journal of Control, 25(4), (1977), pp. 609-620.

[18] T. Kobylarz , I. M. Horowitz and C. H. Houpis, "Flight controller design for uncertain and nonlinear plants with pilot compensation", International Journal of Robust and Nonlinear Control, 2(3), (1992), pp. 183-209.

[19] G. Mateos-Aparicio Morales, "Métodos de interpolación y ajuste. Elementos de matemática actuarial", UCM, ISBN 9788493811204, Madrid. www.ucm.es/info/sevipres/P4/01/ANEXOS01.php, (2010)

[20] P. S. V. Nataraj, "Computation of QFT bounds for robust tracking specifications", Automática 38, (2002), pp 327-334.

[21] K. Ogata, "Ingeniería de control moderna”, Prentice Hall, (1993)

[22] Translation, http://es.mathworks.com/matlabcentral/fileexchange/59068-specifications-translation, (2016)

[23] S. F. Wu, M. J. Grimble and S.G. Breslin, "Introduction to quantitative feedback theory for lateral robust flight control systems design", Control Engineering Practice 6, (1998), pp. 805-828.

[24] O. Yaniv, "Quantitative feedback design of linear and nonlinear control systems", Kluwer Academic Publishers, Norwell, Massachusetts, (1999)

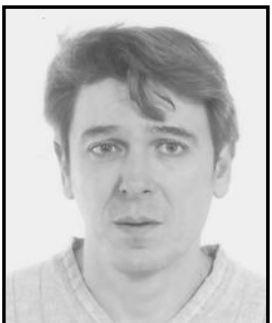

Javier Joglar-Alcubilla works as a professor in the avionics department of the Barajas Institute in Madrid, Spain. He received his B.Sc in Aeronautics Engineering from Polytechnic University at Madrid, Spain, in 1990 and his M.Sc degree in Electronics and Automation Physics from UNED, Spain, in 1994. He received his $\mathrm{Ph} . \mathrm{D}$. degree in Informatics Engineering from ETSII at UNED, Spain, in 2015. He is the author of nine technical books. 